\title{
Universality classes in the random-storage sandpile model
}

\author{
Alexei Vázquez ${ }^{1}$ and Oscar Sotolongo-Costa ${ }^{1,2}$ \\ ${ }^{1}$ Department of Theoretical Physics, Faculty of Physics, Havana University, Havana 10400, Cuba \\ ${ }^{2}$ LICTDI, Faculty of Sciences, UNED, Madrid 28080, Spain
}

\begin{abstract}
The avalanche statistics in a stochastic sandpile model where toppling takes place with a probability $p$ is investigated. The limiting case $p=1$ corresponds to the Bak-Tang-Wiesenfeld (BTW) model with deterministic toppling rule. Based on the moment analysis of the distribution of avalanche sizes we conclude that for $0<p<p_{c}$ the model belongs to the DP universality class while for $p_{c}<p<1$ it belongs to the BTW universality class, where $p_{c}$ is identified with the critical probability for directed percolation in the corresponding lattice.
\end{abstract}

64.60.Lx, 05.70.Ln

Sandpile automata were proposed as a paradigm of self-organized critical (SOC) phenomena [1]. These simple models capture its essential dynamics, which takes place in the form of avalanches of all sizes. At the early state of SOC theory it was believed that the critical state of sandpile automata is insensitive to changes in model parameters, however some recent works contradict this statement. For instance, Vespignani and Zapperi [2] have shown that driving and dissipation rates actually act as control parameters, criticality is obtained after fine tuning of these fields. On the other hand, we have recently shown that a class of models with stochastic rules display a transition from SOC to directed percolation (DP) with increasing the degree of stochasticity [3]. Nevertheless, before we make our final conclusion, we have to investigate if the original Bak-Tang-Wiesenfeld (BTW) sandpile automaton and these modified models belong to the same universality class, otherwise they would just be different models. One may thus ask: do deterministic and stochastic sandpile models belong to the same universality class?

The BTW automaton is defined on $d$-dimensional hypercubic lattice of linear size $L$. On each site $i$ an integer variable $z_{i}$ is defined, which we call energy following [2]. Energy is added to the system by selecting a site at random and increasing its energy by one, i.e. $z_{i} \rightarrow z_{1}+1$. The energy addition continues until one of the sites reaches or exceeds a threshold $z_{c}=2 d$, then this site topples transferring energy to its neighbors. Toppling is defined by the set of rules $z_{i} \rightarrow z_{i}-z_{c}$ and $z_{j} \rightarrow z_{j}+1$ at each of the $j$ nearest neighbors. The toppling at one site may induce an avalanche of toppling events. After the avalanche has stopped we re-start adding energy to the system. Open boundary conditions are assumed. The toppling rules of this model are deterministic and the only randomness is introduced through the random energy addition.

May be the simplest stochastic sandpile model is the Manna or $d$-state model 昍. In this case $z_{c}=d<2 d$ and, therefore, only $d$ of the $2 d$ neighbors will receive energy after toppling. These $d$ neighbors are selected at random. Real-space renormalization group approach [5] suggests that the BTW and Manna models belongs to the same universality class. However, there is not complete agreement in numerical simulations. Early large scale simulations of the Manna [4] and BTW models [6] in two dimensions, show that the avalanche distributions are described by the same exponents for the power law decay and the scaling of the cutoffs. These results where supported by more recent estimates of the avalanche exponents by Lübeck and Usadel [7]. On the contrary, Ben Hur and Biham [8] analyzed the scaling of conditional expectation values of various quantities, obtaining significant differences in the exponents for the two models. However, Chessa et al have recently shown [9] that the method of conditional expectation values, introduced by Ben Hur and Biham, is systematically biased by nonuniversal corrections and, therefore, does not provide indications on universality classes. Moreover, according to the large scale simulations performed by Chessa at al [9], the BTW and Manna models belong to the same universality class.

In the Manna model the randomness appears in the energy transfer after toppling, but the condition for toppling is deterministic. Motivated on a directed model by Tádic and Dhar 10 we have proposed a different model, where sites topples when $z \geq z_{c}=2 d$ but with probability $p$ [3]. In this case, as in the BTW model, each of the $2 d$ neighbors receives one energy grain but the condition for toppling is stochastic. In the particular case $p=1$ one recovers the BTW model. For $p<1$ sites accumulate a random amount of energy, random storage model (RSM) 11]. From mean-field theory and numerical simulations in one dimension [3] we obtain that the RSM self-organizes into a critical state for $p_{c}<p<1$, while it is subcritical for $p<p_{c}$. The subcritical state is found to be similar to DP and $p_{c}$ is identified with the critical threshold for DP. The correlation length exponents are identical to those of DP but other exponents result different due to open boundary conditions. However, in that occasion our analysis was limited to a small region close to $p_{c}$ and simulations were performed only in $d=1$. Thus, we could not provide any indication about the universality classes. 
In the present work we extend the numerical simulations of the RSM to the whole range of $p$, in one and two dimensions. To determine the avalanche size and duration exponents we use moment analysis, a technique previously introduced by de Menech at al [12] to obtain the scaling exponents for the BTW model. techniques. The numerical evidence suggests that the RSM and the BTW model belong to different universality classes. Moreover, for $p<p_{c}$ we corroborate that the model is similar to DP.

The MF theory of the RSM [3] reveals that there is a critical probability $p_{c}$ below which the system is subcritical. In this regime $p$ is small and sites accumulates a large amount of grains. Let us assume that all sites have heights above the critical threshold $z_{c}$. Then when a new grain is added at certain site this site will topples with probability $p$. If it topples then each of its nearest neighbors will also topple with probability $p$ and so on. This picture is equivalent to a site directed percolation problem, where sites become active with probability $p$ if one of its neighbors was active in the previous step. Hence the correlation length is given by $\xi \sim\left(p_{c}-p\right)^{-\nu}$, where $p_{c}$ is the critical threshold for site directed percolation in the corresponding lattice and $\nu$ is the spatial correlation length exponent. Now, if $p \ll p_{c}$ in such a way that $\xi \ll L$, where $L$ is the system size, then an avalanche starting from a bulk site will never reach the boundary and, therefore, no grain will be dissipated. Hence, the average height will increase in time because dissipation will no balance the grain addition from the external field. After a time long enough most sites will have heights above $z_{c}$, supporting our starting assumption. The dynamical state below $p_{c}$ is thus equivalent to DP and $p_{c}$ is identified with the site DP threshold in the corresponding lattice. Above $p_{c}$ and for $z>z_{c}$ at all sites the system will still be equivalent (at least for small times) to DP and an infinite avalanche will be generated. However, such state is not stable because the infinite avalanche will reach the boundary leading to dissipation of grains and hence the decrease of the column heights up to the avalanche has stopped. Above $p_{c}$ the stationary state is no more equivalent to DP. The balance between the grains added by the external field and dissipation at the boundary leads to a SOC state. Our task will be to determine if this SOC state belongs to the same universality class of the BTW model.

Let $s$ be the avalanche size and $T$ its duration. $s$ is the number of toppling events in an avalanche. $T$ is the number of steps required to obtain a stable configuration starting from the initial site with $z \geq z_{c}$, which triggered the avalanche, taking into account that on each step all sites are updated in parallel following the toppling rule. Both, $s$ and $T$, are random variables. Their distributions are given by, assuming scaling,

$$
P_{x}(x)=x^{-\tau_{x}} f_{x}\left(x / x_{c}\right),
$$

where $x$ is $s$ or $T, s_{c}$ and $T_{c}$ are the cutoff avalanche size and duration, and $f_{x}$ is a cutoff function. The cutoffs scale with the characteristic length of the system $\xi$ according to

$$
x_{c} \sim \xi^{\beta_{x}}
$$

where $\beta_{s}=D, \beta_{t}=z, D$ is the avalanche dimension and $z$ the dynamic scaling exponent. In the SOC state $\xi \sim L$ and, therefore, the cutoffs scale with system size. On the contrary, in the subcritical state $\xi \sim\left(p_{c}-p\right)^{-\nu}$ and, hence, the cutoffs diverges when $p$ approaches $p_{c}$. The scaling exponents $\tau_{x}$ and $\beta_{x}$ are not all independent. From the identity $P(s) d s=P(T) d t$ one obtains

$$
\left(\tau_{s}-1\right) D=\left(\tau_{t}-1\right) z .
$$

On the other hand, in the SOC state $\left(p>p_{c}\right)$ the mean avalanche size should scale as $\langle s\rangle \sim L^{2}$ and, therefore,

$$
\left(2-\tau_{s}\right) D=2 .
$$

These scaling relations are useful to test the reliability of the numerical estimates.

The purpose of present numerical simulations is to determine the exponents $\tau_{s}, \tau_{t}, D$ and $z$. We are going to take up this task using as fundamental technique the moment analysis [12]. The $q$ moment is given by

$$
\left\langle x^{q}\right\rangle=\int d x P(x) x^{q} \sim \xi^{\sigma_{x}(q)},
$$

where

$$
\sigma_{x}(q)=\beta_{x}\left(1-\tau_{x}\right)+\beta_{x} q
$$

The last equivalence in eq. (5) is not valid for small values of $q$. For small $q$ the integral depends in the functional form of $P(x)$ in the whole range of $x$, while scaling assumptions are in general not valid for small $x$. The extreme case is $q=0$, normalization imposes $\sigma_{x}(0)=0$ and, therefore, eq. (6) is not valid. But, for $q \geq \tau_{x}-1$ large $x$ dominates leading to the last equivalence in eq. (5).

After computing the moments one can obtain $\sigma_{x}(q)$ from a liner fit to the log-log plot of $\left\langle x^{q}\right\rangle$ vs. $\xi$. Then one can obtain $\tau_{x}$ and $\beta_{x}$ from a linear fit to the straight part of the plot $\sigma_{x}(q)$ vs. $q$. Above $p_{c}$ we have $\xi \sim L$, but it is a function of $p$ below. To compute the correlation lenght below $p_{c}$ we use the following expression [3]

$$
\xi^{2} \sim \frac{\sum_{t=0}^{\infty} \sum_{i=0}^{L}\left(i-i_{0}\right)^{2} \rho_{a i}}{\sum_{t=0}^{\infty} \sum_{i=0}^{L} \rho_{a i}},
$$

where $i_{0}$ is the position of the initial active $\left(z \geq z_{c}\right)$ site, $t$ is the number of steps measured in the time scale of the avalanche and $\rho_{a i}=1\left(\rho_{a i}=0\right)$ in active (inactive) sites. Moreover, from the log-log plot of $\xi$ vs. $p_{c}-p$ one can obtain a numerical estimate of $p_{c}$ and $\nu$.

$d=1$ : The BTW model $(p=1)$ in one dimension exhibits trivial critical behavior [1]. No power law behavior 
is observed in the avalanche size and duration distributions, however one can compute the exponents $D$ and $z$ from the scaling of the moments with system size, result$\operatorname{ing} D \approx 2$ and $z \approx 1$. Using this values and the scaling relations in eqs (3) and (4) one could obtain the exponents $\tau_{s}$ and $\tau_{t}\left(\tau_{s}=\tau_{t}=1\right)$ however these scaling laws are not valid in this case because the distributions of avalanche size and duration do not follow the scaling law in eq. (11). On the contrary, the RSM in one dimension has non trivial behavior. The $q$ dependence of $\sigma_{s}$ and $\sigma_{t}$, for different values of $p<1$, is shown in figs. 11 and 2, respectively. The numerical estimates of the scaling exponents are given in table If For $p_{c}<p \leq 0.8$ we observe that $\sigma_{s}(q), D, \tau_{s}$ and $\tau_{t}$ are practically insensitive to changes in $p$, but systematic deviations are observed for $\sigma_{t}(q)$ and $z$. For $p=0.9$ the scaling exponents are between those for $p=1$ and $p=0.8$. On the other hand, using the numerical data below $p_{c}$, we have obtained $p_{c}=0.707 \pm 0.002$, $\nu=1.07 \pm 0.03$ and $z=1.57 \pm 0.02$ which are, within the numerical error, identical to the series expansion estimates for site DP in a square lattice 13. Moreover, the exponents $\delta=\tau_{t}-1$ and $z$ are consistent with previous numerical simulations [3]. We have also carry out finite size scaling of the distributions of avalanche size and duration. In all cases, including the BTW limit, we observe a good data collapse and the obtained scaling exponents are in agreement with those obtained from the moment analysis. Moreover, within the numerical error, the scaling relations in eqs. (3) and (4 are satisfied.

$d=2$ : The BTW model has nontrivial exponents. However, the data collapse was not compatible with the scaling assumption in eq. (11). The corrections to scaling in the BTW has been found to be very strong [7,9], making necessary the use of very large lattice sizes to obtain accurate estimates of the scaling exponents. The largest lattice size used in our simulations, $L=512$, seems to be not large enough. Using lattice sizes ranging from $L=512$ to $L=2048$ Chessa et al [9] have obtained a good finite size scaling for the distribution of avalanche size, but have not for the distribution of avalanche duration. We thus rule out the possibility of determine the BTW exponents in two dimensions with such small lattice sizes. Instead of that, we are going to use the numerical estimates by Chessa et al [9] and Lübeck and Usadel [7]. On the contrary, the RSM displays good finite size scaling for the lattice sizes we have used. Moreover, the scaling exponents obtained from the finite size scaling are in agreement with those obtained from the moment analysis. The $q$ dependence of $\sigma_{s}(q)$ and $\sigma_{t}(q)$ is shown in figs. 3 and 4, respectively. The numerical estimates of the scaling exponents for different values of $p<1$ are given in tab. II, toguether with the reports by Chessa et al [9] and Lübeck and Usadel [7] for the BTW model. $\sigma_{s}(q), D, \tau_{s}$ and $\tau_{t}$ are practically insensitive to changes in $p$ above $p_{c}$, even considering the deterministic limit. On the contrary, $\sigma_{t}(q)$ and $z$ suffer from systematic deviations with changing $p$. In this case we must be more careful because the finite size effects have stronger in- fluence on the distribution of avalanche duration. For instance, for the largest lattice size used $L=512$ the distribution of avalanche sizes cover about six decades while the distribution of avalanche durations cover less than five decades. To obtain more precise determination of the dynamic scaling exponents we must increase system size. In the mean time, the scaling exponents of the distribution of avalanche sizes indicates that the RSM in the range $p_{c}<p<1$ belongs to the same universality class of the BTW model, which correspond with the limit $p=1$. On the other hand, below $p_{c}$ we have obtained $p_{c}=0.344 \pm 0.001, \nu=0.728 \pm 0.002$ and $z=1.76 \pm 0.02$ which are, within the numerical error, identical to the numerical estimates for site DP in a BCC lattice 14. In this case the difference between the exponents below and above $p_{c}$ is significant, showing that the RSM above and below $p_{c}$ belongs to different universality classes.

The numerical simulations in $d=2$ corroborate that the RSM is similar to DP below $p_{c}$. As in $d=1$, the exponents $\nu$ and $z$ and the critical probability $p_{c}$ are identical to the DP values in the corresponding lattice. On the other hand, the difference between the exponents $D$ and $z$, obtained using the data below $p_{c}$ and those obtained above but close to $p_{c}$, is far form being contained within the error bars. The model above and below $p_{c}$ belong to different universality classes. Below $p_{c}$ it is DP with open boundaries and random initial seed, while above $p_{c}$ we will continue using the term RSM.

The numerical evidence obtained for the avalanche size distribution indicates that in $d=2$ the RSM belong to the universality class of the BTW model. The scaling exponents $\tau_{s}, \tau_{t}$ and $D$ are practically independent of $p$ in the SOC regime $p_{c}<p \leq 1$, however, $z$ shows a strong $p$ dependence which may be attributed to finite size effects. On the contrary in $d=1$ the distribution of avalanche sizes and duration for the BTW model display trivial behavior while in the RSM they satisfy the scaling hypothesis. In larger dimensions $d>2$ we expect that the SOC regime of the RSM belongs to the BTW universality class as in $d=2$.

In summary the RSM has three different regimes. I: $0<p<p_{c}$ similar to DP, II: $p_{c}<p<1$ where the toppling rule are still stochastic but the system is in a SOC state and III: $p=1$ (BTW) the toppling rules are deterministic. Based on the moment analysis of the distribution of avalanche sizes we conclude that for $0<p<p_{c}$ the model belongs to the DP universality class while for $p_{c}<p<1$ it belongs to the BTW universality class.

We thanks A. Vespignani for bringing up our attention on two-dimensional simulations. This work was partially supported by the Alma Mater prize, given by the University of Havana. 
[1] P. Bak, C. Tang, and K. Wiesenfeld, Phys. Rev. Lett. 59, 381 (1987); Phys. Rev. A 38, 364 (1988).

[2] A. Vespignani and S. Zapperi, Phys. Rev. Lett. 78, 4793 (1997); Phys. Rev. E 57, 6345 (1998).

[3] A. Vázquez and O. Sotolongo-Costa, cond-mat/9810408 (submitted to J. Phys. A).

[4] S. S. Manna, Physica A 179, 249 (1991).

[5] L. Pietronero, A. Vespignani, S. Zapperi, Phys. Rev. Lett. 57, 1690 (1994); A. Vespignani, S. Zapperi, and L. Pietronero, Phys. Rev. E 51, 1711 (1995).

[6] P. Grassberger and S. S. Manna, J. Phys. (France) 51, 1077 (1990); S. S. Manna, J. Stat. Phys. 59, 509 (1990); Physica A 179, 249 (1991).

[7] S. Lübeck and K. Usadel, Phys. Rev. E 55, 4095 (1997).

[8] A. Ben-Hur and O. Biham, Phys. Rev. E 53, R1317 (1996).

[9] A. Chessa, H. E. Stanley, A. Vespignani, and S. Zapperi, cond-mat/9808263.

[10] B. Tádic and D. Dhar, Phys. Rev. Lett. 79, 1519 (1997).

[11] A. Vespignani, personal communication.

[12] M. de Menech, A. L. Stella, and C. Tebaldi, Phys. Rev. E 58, R2677 (1998).

[13] I. Jenssen, J. Phys. A 29, 7013 (1996).

[14] P. Grassberger, J. Phys. A 22, 3673 (1989).

\begin{tabular}{lllll}
\hline \hline$p$ & $D$ & $z$ & $\tau_{s}$ & $\tau_{t}$ \\
\hline 1 & 2 & 1 & & \\
0.9 & $2.25(1)$ & $1.48(2)$ & $1.12(1)$ & $1.17(2)$ \\
0.8 & $2.27(1)$ & $1.52(2)$ & $1.12(1)$ & $1.18(2)$ \\
0.708 & $2.27(1)$ & $1.54(2)$ & $1.13(1)$ & $1.18(2)$ \\
$p<p_{c}$ & $2.34(1)$ & $1.57(2)$ & $1.16(1)$ & $1.20(2)$ \\
\hline \hline
\end{tabular}

TABLE I. Scaling exponents in $d=1$ for different values of $p$.

\begin{tabular}{|c|c|c|c|c|}
\hline$p$ & $D$ & $z$ & $\tau_{s}$ & $\tau_{t}$ \\
\hline$\overline{1}$ & $2.73(2)$ [9] & $1.52(2) 9$ & 1.293 & 1.480 \\
\hline 0.9 & $2.74(1)$ & $1.53(2)$ & $1.28(1)^{\text {b }}$ & $1.48(2)^{0}$ \\
\hline 0.8 & $2.75(1)$ & $1.54(2)$ & $1.29(1)$ & $1.48(2)$ \\
\hline 0.6 & $2.74(1)$ & $1.58^{*}$ & $1.29(1)$ & $1.47^{*}$ \\
\hline 0.4 & $2.74(1)$ & $1.61^{*}$ & $1.28(1)$ & $1.46^{*}$ \\
\hline$p<p_{c}$ & $2.90(1)$ & $1.76(2)$ & $1.27(1)$ & $1.43(2)$ \\
\hline
\end{tabular}

TABLE II. Scaling exponents in $d=2$ for different values of $p$. * These exponents may be affected by finite size corrections. 


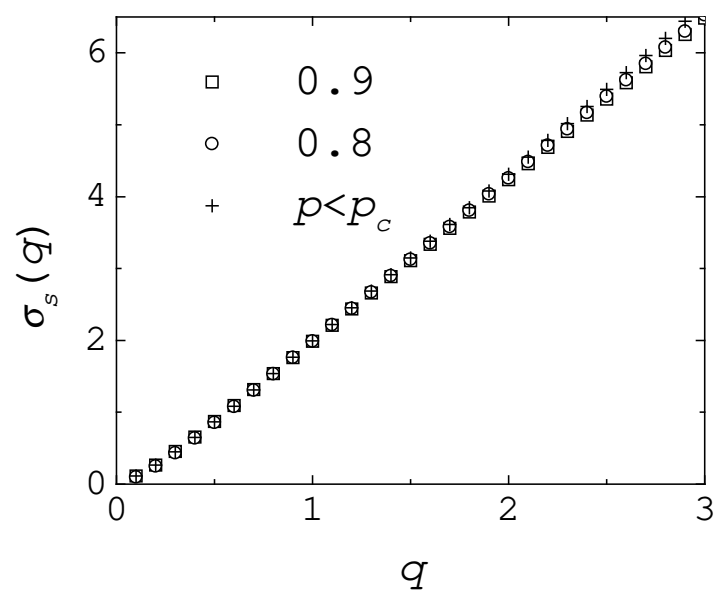

FIG. 1. Plot of $\sigma_{s}(q)$ in $d=1$ for different values of $p$. Data above $p_{c}=0.707(2)$ where obtained using lattice sizes $L=80,160,320$ and 640 . Data below $p_{c}$ where obtained using probabilities $p=0.670,0.688,0.696$ and 0.7000 .

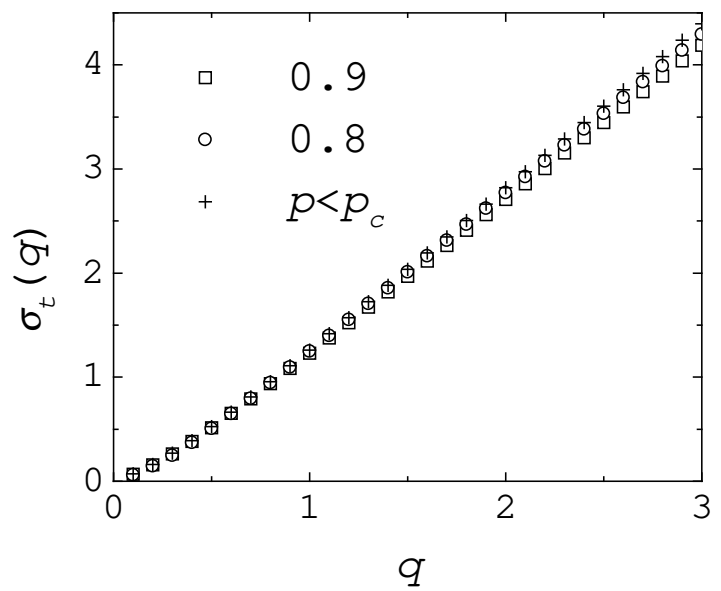

FIG. 2. Plot of $\sigma_{t}(q)$ in $d=1$. The lattice sites and probabilities used are the same as described in the caption of fig. 1.

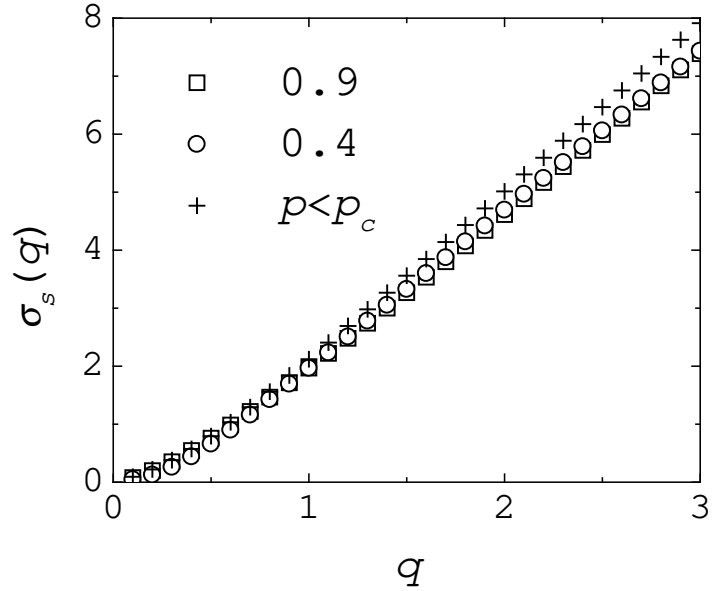

FIG. 3. Plot of $\sigma_{s}(q)$ in $d=2$ for different values of $p$. Data above $p_{c}=0.344(1)$ where obtained using lattice sizes $L=64,128,256$ and 512. Data below $p_{c}$ where obtained using probabilities $p=0.26,0.30,0.33$ and 0.34 .

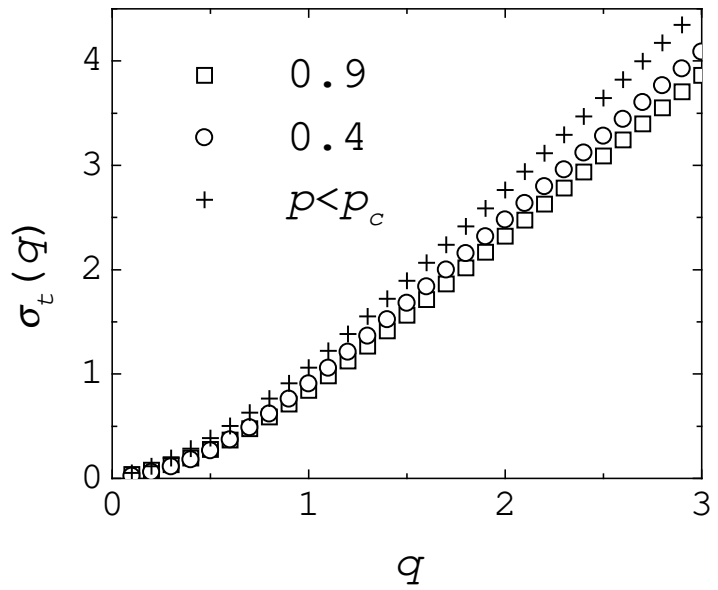

FIG. 4. Plot of $\sigma_{t}(q)$ in $d=2$. The lattice sites and probabilities used are the same as described in the caption of fig. 3. 